\title{
33. NONGRAVITATIONAL FORCES AND PERIODIC COMET GIACOBINI-ZINNER
}

\author{
D. K. YEOMANS \\ Astronomy Program, University of Maryland, College Park, Md., U.S.A.
}

\begin{abstract}
Observations of $\mathrm{P} / \mathrm{Giacobini}$-Zinner could not be successfully represented for more than two successive apparitions using Newtonian equations of motion. The comet's motion has a secular deceleration approximately equal to 0.08 day per (period) ${ }^{2}$. After extending the differential correction to include nongravitational terms in the equations of motion the observations were successfully represented over the time intervals 1900-1946, 1913-1959, and 1939-1965. There is an indication that the magnitude of the nongravitational forces increases with time and that the motion of the comet was discontinuous between 1959 and 1965. A Giacobinid meteor shower prediction has been provided for the 1972 return.
\end{abstract}

Short-period comet Giacobini-Zinner was discovered by M. Giacobini at Nice in 1900 and accidentally rediscovered in 1913 by E. Zinner. It has been observed at eight passages through perihelion and missed altogether at three. Debris from $\mathrm{P} /$ Giacobini-Zinner is believed responsible for the Giacobinid (Draconid) meteor showers. An investigation of this comet's motion was undertaken to determine the role of nongravitational forces.

The numerical integration employed is a predictor-corrector technique running at one-day time steps throughout the comet's orbit. Planetary and solar coordinates are read from magnetic tapes. The differential correction is a 'variation of orbits' procedure and is similar to that used by Marsden (1968). The numerical integration, formation of residuals, and differential correction procedure was programmed for the IBM 360/95 computer at Goddard Space Flight Center.

The first step in determining an orbit for this comet was an attempt to link the successive 1933, 1939 and 1946 apparitions using strictly Newtonian equations of motion. However, after the differential correction routine had converged, the mean residual was nearly 2"12 and the two 1939 observations had residuals nearly equal to $27^{\prime \prime}$. Attempting to link the 1946, 1959, and 1965 apparitions met with even less success. The mean residual was $11^{\prime \prime} .48$ and the five 1965 observations had residuals close to 190 ". Newtonian orbits were then successfully computed for pairs of consecutive apparitions, and a comparison of the predicted and observed times of perihelion passage revealed a secular deceleration in this comet's mean motion approximately equal to 0.08 day per (period) ${ }^{2}$. As a check upon the present work, it was noted that the Newtonian orbits computed for the 1933, 1939 and 1946 apparitions agree with the earlier results of Evdokimov (1963).

In an attempt to represent the observations for more than two apparitions, three nongravitational terms were added to the Newtonian equations of motion. The form of these orthogonal terms is that used by Marsden $(1969,1972)$. The differential correction scheme was extended to include the unknowns $A_{i}$ and $B_{i}$. Generally $A_{1}$ 
and $A_{2}$ were sufficient to provide a good representation of the observations for four or five apparitions, while the inclusion of $B_{2}$ allowed a satisfactory representation for up to six apparitions. Inclusion of a fourth nongravitational parameter $\left(A_{3}\right.$ or $\left.B_{1}\right)$ had a negligible effect upon the results. Ultimately, it was possible to represent successfully the observations over the intervals 1913-1959, 1900-1946 and 1939-1965; see Table I.

TABLE I

Osculating elements and nongravitational parameters (with probable errors)

\begin{tabular}{|c|c|c|c|}
\hline $\begin{array}{l}\text { Interval } \\
\text { Mean resid. } \\
\text { Epoch (ET) }\end{array}$ & $\begin{array}{l}1913-1959 \\
1^{\prime \prime} 22 \\
1913 \text { Oct. } 10.0\end{array}$ & $\begin{array}{l}1900-1946 \\
1^{\prime \prime 4} 48 \\
1900 \text { Dec. } 7.0\end{array}$ & $\begin{array}{l}1939-1965 \\
0 \text {.94 } \\
1939 \text { Aug. } 25.0\end{array}$ \\
\hline$T(\mathrm{ET})$ & $\begin{aligned} 1913 \text { Nov. } 2.56654 \\
\pm 0.00013\end{aligned}$ & $\begin{aligned} 1900 \text { Nov. } 28.49641 \\
\pm 0.00023\end{aligned}$ & $\begin{aligned} 1940 \text { Feb. } & 17.21648 \\
& \pm 0.00071\end{aligned}$ \\
\hline$e$ & $0.7206115 \pm 0.0000001$ & $0.7315675 \pm 0.0000002$ & $0.7166567 \pm 0.0000001$ \\
\hline$q(\mathrm{AU})$ & $0.9760088 \pm 0.0000003$ & $0.9315211 \pm 0.0000005$ & $0.9956677 \pm 0.0000003$ \\
\hline$\Omega^{\circ}(1950.0)$ & $196.36633 \pm 0.00006$ & $197.43252 \pm 0.00008$ & $196.24967 \pm 0.00006$ \\
\hline$\omega^{\circ}(1950.0)$ & \pm 0.00017 & 171.05086 & 171.79083 \\
\hline$i^{\circ}(1950.0)$ & \pm 0.00003 & \pm 0.00006 & \pm 0.00003 \\
\hline$A_{1} \times 10^{5}$ & \pm 0.0175 & +0.5043 & +0.8226 \\
\hline$A_{2} \times 10^{6}$ & \pm 0.0013 & +1.1340 & +0.3567 \\
\hline$B_{2}$ & \pm 0.0012 & \pm 0.0018 & -3.6586 \\
\hline
\end{tabular}

Table II represents successful orbits using different nongravitational parameters. Each orbit was differentially corrected over the time interval indicated, solving for the given nongravitational parameters and their probable errors. The mean residual ('Resid.') for each orbit is listed above the number of observations employed ('Obs.'), and the epoch of osculation appears under the observed interval. Each orbit was integrated forward and backward in time to determine the maximum residuals (in seconds of arc) for the apparitions not included in the differential correction. The units associated with the accelerations are AU per (40 ephemeris days) ${ }^{2}$. Orbits No. 8, 5, and 12 are the ones listed in Table I.

Assuming an icy-conglomerate model for the cometary nucleus (Whipple, 1950), a number of results are evident from Table II:

(1) The radial component of the nongravitational force is directed away from the Sun $\left(A_{1}>0\right)$. The transverse component is directed $90^{\circ}$ forward of the radial component $\left(A_{2}>0\right)$, implying a direct rotation of the nucleus.

(2) There is no evidence for a significant nongravitational force component normal to the orbit plane.

(3) The ratio $A_{2} / A_{1}$ implies a small lag angle $\left(<15^{\circ}\right)$ between the subsolar meridian and the direction of maximum mass ejection.

(4) The magnitude of the transverse component of the nongravitational acceleration is increasing with time over the observed time interval $\left(B_{2}<0\right)$. 
NONGRAVITATIONAL FORCES AND PERIODIC COMET GIACOBINI-ZINNER

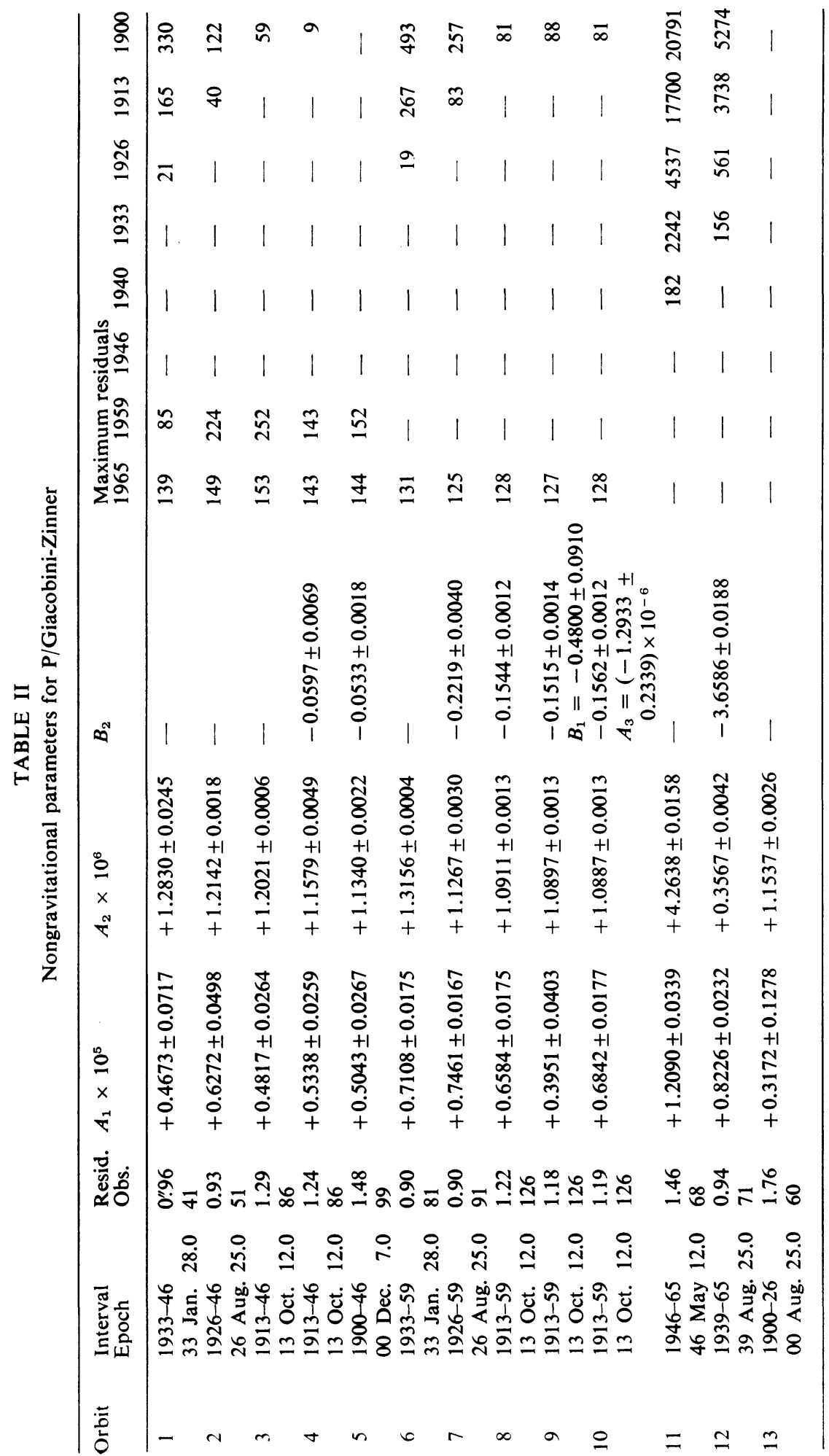


(5) Orbit No. 5 (1900-1946) and orbit No. 8 (1913-1959) both spanned six apparitions, whereas orbit No. 12 (1939-1965) successfully represented the observations of only four apparitions.

The last two points require some elaboration. If $B_{2}$ is negative, $\mathrm{P} /$ Giacobini-Zinner would be the first short-period comet to exhibit an increase in the transverse component of the nongravitational acceleration with time. Independent evidence that this is indeed the case comes from a comparison of the increasing values of $A_{2}$ from orbits No. 13 and 6 in Table II. Although the sign of $B_{2}$ is likely to be negative in all orbits in which it was satisfactorily determined, the magnitude of $B_{2}$ in orbit No. 12 is quite suspicious. It is only with the inclusion of the 1965 observations that the magnitude of $B_{2}$ increases 24 times compared to orbit No. 8 .

In terms of the icy-conglomerate model, two processes come to mind for a secular time increase in the transverse component of nongravitational acceleration. First, if the comet nucleus had an ice mantle, the effective nongravitational force would increase with time as the comet radius decreased. Secondly, one could imagine the magnitude of the outgassing to remain constant while the inclination of the rotation axis with respect to the orbit plane slowly 'straightened up' from an initially inclined position.

The negative value of $B_{2}$ for $\mathrm{P} /$ Giacobini-Zinner is not the only surprise concerning this comet's behavior. The motion of the comet between 1959 and 1965 appears to be discontinuous. The apparent discontinuous behavior is suggested by the results presented in Table II. Orbit No. 8, which successfully represented the observations in 1913,1926, 1933, 1939, 1946 and 1959, produced residuals of approximately $2^{\prime}$ in 1965. The comet was nearly $3 \mathrm{AU}$ from the Earth when observed in 1965, and orbit No. 8 required a correction to the perihelion passage time of $\Delta T=+0.3$. There is no obvious reason why such a large $\Delta T$ correction should be necessary in 1965 . The required $\Delta T$ correction in 1965 is apparently due to an enhancement of the nongravitational forces between 1959 and 1965. The effect could be due to a sudden increase in the comet's orbital velocity. Marsden $(1969,1970)$ has noted that some comets appear to have discontinuous changes in their nongravitational forces following a close approach to Jupiter. Although $\mathrm{P} /$ Giacobini-Zinner had a moderately close approach (0.93 AU) to Jupiter in 1958, there was no close approach between 1959 and 1965 . A case could be made for the splitting of this comet between 1959 and 1965. The 1959 observations showed no evidence of splitting, while the 1965 observations were made near the limit of visibility. The brightest and faintest 1965 observed magnitudes were 19.7 and 20.5 , so that a slightly fainter fragment could easily have been missed. For example, the discrepancy in the 1965 perihelion passage time could be explained if the comet split while approaching aphelion ( $r=5 \mathrm{AU})$ in 1962 and the larger fragment received a sudden increase in velocity of $1.4 \mathrm{~m} \mathrm{~s}^{-1}$. Table III lists osculating elements for epochs near the perihelion times 1900-1966.

Finally, a word about the Giacobinid meteor showers is in order. The Giacobinid meteor showers have probably been the most spectacular meteor displays of the present century. The showers were particularly strong in 1933 and 1946, with lesser showers being observed in 1926 and 1952. The duration of the showers is remarkably 
TABLE III

Osculating elements for epochs near passages through perihelion

\begin{tabular}{cccc}
\hline 1900 Dec. 7.0 & 1907 May 25.0 & 1913 Nov. 9.0 & 1920 May 16.0 \\
1900 Nov. 28.49641 & 1907 May 19.50678 & 1913 Nov. 2.56656 & 1920 May 18.89607 \\
0.7315675 & 0.7317000 & 0.7206140 & 0.7199385 \\
0.9315211 & 0.9308234 & 0.9760089 & 0.9799927 \\
197.43252 & 197.36299 & 196.36625 & 196.31669 \\
171.05086 & 171.12828 & 171.48637 & 171.54205 \\
29.83658 & 29.85905 & 30.74721 & 30.71705 \\
& & & \\
1926 Dec. 31.0 & 1933 July 27.0 & 1940 Feb. 21.0 & 1946 Sept. 17.0 \\
1926 Dec. 11.70840 & 1933 July 15.14737 & 1940 Feb. 17.21100 & 1946 Sept. 18.48600 \\
0.7170052 & 0.7159931 & 0.7167041 & 0.7166791 \\
0.9937348 & 0.9995269 & 0.9956051 & 0.9957057 \\
196.24237 & 196.24296 & 196.24976 & 196.29323 \\
171.75492 & 171.77146 & 171.79206 & 171.81054 \\
30.73865 & 30.68389 & 30.74136 & 30.72686 \\
& & & \\
1953 April 13.0 & 1959 Nov. 8.0 & 1966 Mar. 16.0 & \\
1953 April 16.91679 & 1959 Oct. 26.91926 & 1966 Mar. 28.29078 & \\
0.7178923 & 0.7289474 & 0.7293999 & \\
0.9886907 & 0.9359554 & 0.9335067 & \\
196.23984 & 196.02990 & 195.96510 & \\
171.88762 & 172.84433 & 172.91988 & \\
30.82435 & 30.90379 & 30.94417 & \\
\hline
\end{tabular}

For each set of elements, the consecutive lines represent the osculation epoch, time of perihelion passage (ET), eccentricity, perihelion distance (AU), the longitude of the ascending node, argument of perihelion, and the inclination. The three angular elements are in degrees and are referred to the ecliptic and mean equinox of 1950.0 .

The elements for 1900 and 1907 are from orbit No. 5, and those for 1913 to 1959 are from orbit No. 8. The 1966 elements are from an orbit fitted to observations in 1959 and 1965 but adopting the nongravitational parameters of orbit No. 8 .

short, with most of the meteor activity taking place within $1 \mathrm{~h}$. In 1972, the Earth will pass only $0.00074 \mathrm{AU}$ outside the comet's orbit at the descending node. The Earth will follow the comet to the node by 58.5 days, and the prospect of a meteor shower in 1972 Oct. 8.65 UT is quite good.

\section{References}

Evdokimov, Yu. V.: 1963, Astron. Zh. 40, 544.

Marsden, B. G.: 1968, Astron. J. 73, 367.

Marsden, B. G.: 1969, Astron. J. 74, 720.

Marsden, B. G.: 1970, Astron. J. 75, 75.

Marsden, B. G.: 1972, this Symposium, p. 135.

Whipple, F. L.: 1950, Astrophys. J. 111, 375.

\section{Discussion}

F. L. Whipple: The evidence for an increasing nongravitational effect in $\mathrm{P} /$ Giacobini-Zinner and the suggestion of a transition in the nucleus from ices on the outside toward denser earthy material - 
providing a greater time lag in heat transfer - are most intriguing. Confirming evidence may be found in the fact that the related meteor stream contains the extreme examples of low density and fragile materials. On the other hand, the comet may be uniform throughout and near its final dissolution, which suggests that the comet was never a very large one with an asteroidal core.

L. Kresák: Isn't the observed increase in the nongravitational effects only an effect of longperiod variations?

D. K. Yeomans: Yes, I rather think so. 